\title{
TITLE:
}

\section{Critical Conditions for Brittle Fracture of Asphalts in Extension}

$\operatorname{AUTHOR}(S)$ :

Gotoh, Rempei; Aida, Hiroshi

\section{CITATION:}

Gotoh, Rempei ... [et al]. Critical Conditions for Brittle Fracture of Asphalts in Extension. Bulletin of the Institute for Chemical Research, Kyoto University 1961, 39(3): 250-251

ISSUE DATE:

1961-07-31

URL:

http://hdl.handle.net/2433/75829

RIGHT: 


\title{
Studies of the Oxidation of Hydrocarbon Oils by Means of Infrared Absorption Spectra
}

\author{
Pempei Gotoh, Tohru Takenaka and Soichi Hayashi \\ Kogyo Kagaku Zasshi (Journal of the Chemical Society of \\ Japan, Industrial Chemistry Section), 63, 729 (1960)
}

Liquid paraffin was heated at temperatures between $100^{\circ} \mathrm{C}$ and $450^{\circ} \mathrm{C}$, and for less than 450 minutes under three different supplies of gases; (a) bubbling of $\mathrm{N}_{2}$, (b) exposing in air, and (c) bubbling of air. The infrared analysis of the liquid products obtained in the reaction vessel at temperatures higher than $100^{\circ} \mathrm{C}$ showed formation of hydroperoxide, ketone, acid, aldehyde and ester, and at temperatures higher than $300^{\circ} \mathrm{C}$, the absorption bands of three kinds of double bonds, namely, transvinylene, vinyl and vinylidene groups were observed. The dependence of the amount of the oxygenated group and double bond formation on the heating condition, temperature and time was observed, and compared with the results which have been reported on the effect of irradiation of hydrocarbons with various radiation of different linear energy transfer. Gaseous products trapped by the freezing mixture of dry ice-ethanol consisted of acetone, acetaldehyde and unsaturated molecules which were mainly $n$-bntylene (1) and isobutylene, whereas those trapped by liquid air consisted of carbon monoxide, carbon dioxide, methane and ethylene, The amount of these gaseous products increased linearly with the increase of heating temperature, except acetone and acetaldehyde which were saturated at a certain temperature.

\section{Critical Conditions for Brittle Fracture of Asphalts in Extension}

\author{
Rempei Gotoh and Hiroshi AidA \\ Zairyo Shiken (Journal of the Japan Society for \\ Testing Materials), 9, 331 (1960)
}

The change in the tensile force of several kinds of blown asphalts was recorded at various temperatures and rates of extension. The critical velocity for brittle fracture, $V_{B}$, was defined by discontinuous break-down of the tensile force followed by no deformation. The relation between $V_{B}$ and the temperature, $T$, was given empirically by a relation as follows:

$$
V_{B}=A_{\text {exp }}\left(-E_{B} / R T\right)
$$

where $\mathrm{A}$ is an experimental constant, $\mathrm{E}_{\mathrm{B}}$ the apparent activation energy and $R$ the gas constant.

It was assumed that brittle fracture occurs when the viscous resistance exceeds the cohesion of the viscous materials locally. Following the rate process theory of viscous flow and from the relation (1), we have 


$$
E_{B}-R T \ln A=\Delta F^{*}-R T \ln K,
$$

where $\Delta F^{*}$ is the free energy of activation for flow and $K$ a constant which depends upon the shear stress, the temperature and the vibrational frequencies of the liquid molecules concerned. Though neithcr $\Delta F^{*}$ and $K$ can be evaluated directly, the left side of the relation (2) can be estimated experimentally and it may give the measure relative to $\Delta \mathrm{F}^{*}$. It was shown that the linear relations between $\log V_{i}$ and $1 / T$ shift according to values of $\left(E_{B}-R T \ln A\right)$ at a certain temperature which depends upon the composition of the asphalts used.

Effects of colloidally fine fillers on the flow properties of asphalts were observed. It was found that carbon black reduces $V_{B}$ remarkably in comparison with colloidal silica and calcium carbonate.

\section{Spiral Growth of Colloidal Gold and Moiré Fringe \\ Eiji Surto and Natsu UyEdA \\ Nature, 185, 453 (1960)}

Various growth spirals and related matters recently observed by electron microscopy on lamellar single crystals of gold, prepared colloid-chemically under definite conditions as discussed in previous papers.

A small hole exists at the centre of the spiral, from where the ridge line starts and, after passing on the outer perimeter of the crystal, come back to the initial starting point. The two spirals on both surfaces of a crystal are nearly symmetrical to each other in most of the present examples.

In the dark-field image, formed with the $(2 \overline{2} 0)$ reflexion there appeared nearly parallel thick moir'e fringes, the direction of which is again parallel to $[2 \overline{2} 0\rfloor$ axis of the crystal. This means that they were formed by the interaction of two electron beams reflected respectively by each $(2 \overline{2} 0)$ plane contained in two superposed steps.

When the cause of the fringe is supposed to be attributable only to the slight azimuthal rotation of steps, the angle of rotation $\alpha$ is given by an equation: $\alpha=$ $d_{2 \overline{2} 0} / D$, where $D$ and $d_{2 \overline{2} 0}$ are the spacing of the moiré fringe and the interplanar

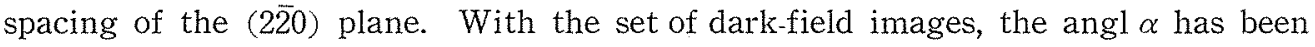
estimated to be of the order of $2 \times 10^{-3}$ red. on an average. Further, it increases roughly linearly from $7 \times 10^{-4}$ red. to $4 \times 10^{-3}$ red. or to much larger values as the position on the step, where the spacing of the fringe was meaured, approaches the centre of the crystal along the spiral step.

As for the small hole, Frank has derived the relationship between the hole diameter $\left(D_{0}\right)$ and the strength of the Burgers vector (b) at the core of the screw dislocation as follows, though slightly modified here:

$$
b=2 \pi\left(S \cdot D_{0} / G\right)^{1 / 2}
$$

where $S$ and $G$ are the surface free energy and modulus of rigidity of the material. The hole diameter is distributed over a range of $200-2400 \mathrm{~A}$. and the most 